\title{
How does verb aspect constrain event representations?
}

\author{
CAROL J. MADDEN and ROLF A. ZWAAN \\ Florida State University, Tallahassee, Florida
}

\begin{abstract}
We investigated the relative contribution of perfective and imperfective aspectual cues on situation models. In Experiment 1, participants were more likely to choose pictures showing completed events than pictures showing ongoing events when they had read perfective sentences, but chose either picture after reading imperfective sentences. In Experiment 2, only one picture was presented and participants were faster to respond to completed pictures than to ongoing pictures when they had read perfective sentences, but showed no latency differences after reading the imperfective sentences. In Experiment 3, participants were faster to read perfective sentences after having seen completed pictures rather than intermediate pictures, but there was no difference for imperfective sentences. The consistent pattern of results demonstrates that readers construct mental representations of completed events when the perfective aspect is used to describe an event. The lack of effect on imperfective sentences and pictures suggests that each reader represents an in-progress event at varying stages of completion.
\end{abstract}

The units of language in narratives are thought to act as processing cues, directing the comprehender's construction of a mental representation of described events (e.g., Givón, 1992). These representations have been called mental simulations (Barsalou, 1999) or situation models (van Dijk \& Kintsch, 1983; Zwaan \& Radvansky, 1998), and they constantly evolve as the comprehender encounters new information. They are thought to incorporate not only the events in a described situation, but also the agents, causality, goals, thoughts, actions, location, and temporal setting involved in the events. Situation models are constructed through a dynamic interaction of pragmatic and grammatical linguistic cues, background knowledge, and episodic information about events narrated previously (previous situation model versions).

Because linguistic cues direct the construction of situation models, subtle changes in syntactic form are assumed to give rise to different mental representations of described situations (Givón, 1992). Skilled language comprehension requires sensitivity to such cues, one of which is verb aspect. Verb aspect is the grammatical construction that denotes an event's duration, onset, and completion status. Verb aspect has received much attention from linguists (Comrie, 1976; Dowty, 1986; ter Meulen, 1995; Vendler, 1957, 1967), yet it lacks empirical investigation. Specifically, there is a lack of empirical research on the impact of subtle verb aspect manipulations on compre-

\footnotetext{
We thank Robert Brush for help in stimulus construction and data collection on this project, as well as Chris Schatschneider for help with the analysis of the data. This research was supported by Grant MH 63972 from the National Institute of Mental Health. Correspondence should be addressed to either C. J. Madden or R. A. Zwaan, Department of Psychology, Florida State University, Tallahassee, FL 32306-1270 (e-mail: madden@psy.fsu.edu or zwaan@psy.fsu.edu).
}

henders' representations. In the present study, we examined the influence of verb aspect on readers' representations of described events.

One simple and broad aspectual category distinction is that of perfectivity (Comrie, 1976; Dowty, 1986; Vendler, 1957). The perfective aspect conveys an outside-theevent perspective, and permits one to view the event as a complete whole. This does not mean that the event does not have any duration. Instead, perfective aspect prevents access to the internal structure of the event. The different stages of an event described in the perfective aspect are not available, only the single whole event. The simple past tense is one way to represent an event in the perfective aspect (e.g., "The boy WALKED to the store."). In this perfective example, the reader would not have access to the various stages of the boy's walk to the store, such as his walking away from his house, his crossing main street, or his arrival at the store. Instead, the reader would simply represent the event as a whole. However, for the event to be represented as a whole, its end must be included, implying that the event was finished. After all, one cannot say, "The boy walked to the store, but he never arrived there."

In contrast, the imperfective aspect provides a withinthe-event perspective, and enables one to view the event as ongoing. Thus, the internal structure, or the various stages of the event, becomes available. Because the imperfective aspect conveys a within-the-event perspective, the onset of the event has occurred before the current point on the narrative time line, but the event has not yet ended. The progressive tense is one means of conveying an event in the imperfective aspect (e.g., "The boy wAS WALKING to the store."). Most often, when the imperfective aspect is used to describe an event, the event is implied to be in a middle stage of completion (Comrie, 1976). 
Thus, in this example, one would assume that the boy was in the process of walking to the store and had not yet reached his destination. It is thus acceptable to say, "The boy was walking to the store, but he never arrived there," which one could not say if the verb were in the perfective aspect. For the purposes of the present study, the simple (e.g., walked) and progressive (e.g., was walking) past tenses were used to convey perfective and imperfective aspect, respectively.

Readers use aspectual cues to help themselves construct representations of events. Presumably, when readers encounter an event described in the perfective aspect, they construct a representation of the event as having been completed. This representation foregrounds the event and its completion, rather than the agent and his efforts to accomplish the task. The event is thus collapsed into a whole, without representing the agent's progress at intermediate stages. Such a representation is more like the experience of remembering an event that has already occurred, without attending to the specific stages that led to its completion. When we remember an event in this way, the collapsed event is foregrounded, and thus, we represent the resulting state.

In contrast, when readers encounter an event described in the imperfective, they construct a representation of the event in progress. Here, the reader represents the internal structure of the event and the agent's progress at intermediate stages. This type of representation foregrounds the agent and his efforts to accomplish the task rather than the complete event itself. Representations of events described in the imperfective are more like actual experiences of ongoing events, rather than like remembering events that have already occurred. When we represent events in this way, the resulting states are less available, and the current state of progress toward completion of the event is foregrounded.

Previous investigations have shown that readers do take aspectual cues into account as they construct situation models of events. For instance, Magliano and Schleich (2000) found, in responses to explicit questions about the completion status of the events, that readers interpreted imperfective actions as ongoing, but perfective actions as completed. This aspectual discrimination interacted with general knowledge about the typical duration of actions.

In addition to these subtle representational influences on event duration and completion, verb aspect is thought to have other functions in language comprehension. It is thought, for instance, to play a role in the foregrounding and backgrounding of information in discourse (Hopper, 1979; Morrow, 1985b). When an event is described in the imperfective aspect, it often serves as a background for events that are described in the perfective aspect, as in "The boy was playing outside when his father arrived." Also, verb aspect is thought to influence the subsequent availability of events, or features of events, in discourse. Carreiras, Carriedo, Alonso, and Fernández (1997) have demonstrated that characters in short narratives are subsequently more accessible when their actions are de- scribed in the past progressive (imperfective) rather than the past perfect (perfective) aspect. Likewise, Ferretti (2000) has demonstrated that the typical locations of events are significantly primed by imperfective verbs, but not by perfective verbs.

Although readers have been shown to use aspectual cues when they construct situation models, the independent influence of the perfective and imperfective aspect has been difficult to examine. The perfective aspect does not allow access to the internal structure of the event, whereas the imperfective aspect does. Therefore, it is much more difficult to assess the activation of perfective event representations than it is to assess the activation of imperfective event representations. It is difficult to probe for the complete event rather than parts of the event or entities involved in the event (i.e., agents and locations), which are only available when the imperfective aspect is used. Thus, probe-word methodologies incorporate a bias for activation of imperfective events because one can probe for entities within the imperfective aspect, which allows access to the internal structure of the event, but not for entities within the perfective aspect. In fact, the use of the perfective aspect has been interpreted as diminishing the overall activation level of an event in the situation model (over time) relative to the imperfective aspect (Magliano \& Schleich, 2000).

In the present study, we investigated the extent to which readers are sensitive to both perfective and imperfective aspect while constructing situation models. We also examined the level of constraint imposed by perfective and imperfective aspects on event representations. We accomplished this through a method of picture presentation, which allowed us to differentially assess the availability of both in-progress and completed events in the situation model in a way that is not afforded by single probe-word presentations. By using events pictured at an intermediate as well as a completed stage, we were able to assess the availability of both imperfective and perfective event representations.

Other researchers have also turned to picture presentations as an alternative to probe words in assessing mental models. For instance, in their landmark experiments, Clark and Chase (1972) used a sentence-picture comparison task to better understand the format and the construction process of readers' representations for described situations. More recently, Glenberg, Robertson, Jansen, and Johnson-Glenberg (1999, Experiment 3) recorded response times to pictured objects presented following negated or positive sentences in isolation. They found that pictures that matched the resulting situation (regardless of negation) were responded to more quickly than pictures that contradicted the described situation, and that pictures following negated sentences elicited slower responses overall. Also, Stanfield and Zwaan (2001) presented pictures following presentation of sentences that implied orientation of objects. Participants were faster to respond to pictures in which the orientation of the object matched that implied by the sentence. In addition, Zwaan, Stanfield, and Yaxley (2002) 
found that participants were faster to respond to pictures in which the shape of the object matched the shape implied by the sentences.

In the present set of experiments, we employed a similar design to investigate the effect of verb aspect on readers' event representations. In Experiments 1 and 2, we examined the nature of aspectual information in event representations formed during the reading of sentences by having readers compare these representations with perceptual information about the events-namely, pictures of the events. In Experiment 3, we examined the representations from the opposite direction. Here, we tested the idea that the aspectual information in a picture of an event can facilitate the subsequent construction and evaluation of an event representation from a sentence.

\section{EXPERIMENT 1}

Experiment 1 was designed to examine readers' sensitivity to aspectual cues. A second goal was to examine potential differences in the extent to which perfective and imperfective aspects constrain the readers' representations of described situations. If perfective and imperfective aspect constructions constrain readers' representations as described in the previous section, participants should form representations of completed events when the events are described in the perfective, and in-progress representations when the events are described in the imperfective.

Participants read sentences and chose one of two pictorial representations of the described action. The sentences were presented in the perfective or imperfective aspect (simple or progressive past tense), and the subsequent pictures showed the event completed as well as in progress (see the Appendix).

Although the perfective aspect does not necessarily emphasize the termination of an action, a finished event was considered to be the best pictorial representation of the event as a single whole. Upon seeing pictures of the completed and the in-progress events, the participants were to choose which picture best matched the sentence. If the perfective aspect constrains readers' representations to completed events, and if the imperfective aspect constrains readers' representations to ongoing events, participants should choose the completed pictures after having read the perfective sentence versions, and the in-progress pictures after having read the imperfective sentence versions.

\section{Method}

Participants. Twenty undergraduate students enrolled at Florida State University participated in the experiment. Participation was part of a course requirement in psychology classes. All participants were native English speakers.

Materials. Twenty-six experimental sentences were constructed, each describing a subject engaging in an action. All actions were events with duration and obligatory temporal end points, such as "driving home." These are called accomplishments (Vendler, 1967), or filters (ter Meulen, 1995). The global description of an accomplishment or filter does not apply to any smaller part of the event. For instance, the smaller part of turning left on Copeland Avenue is not in and of itself "driving home." In contrast, "driving around" is an event with duration, but without an obligatory temporal end point. These types of events are called processes (Vendler, 1967), or holes (ter Meulen, 1995), and they have no specific series of stages leading to an end point. Rather, they can be discontinued at any time. The global description of a process or hole applies to any smaller part of the event. For instance, turning left on Copeland Avenue is, in fact, "driving around." One can finish "driving around" at any point, but one cannot finish "driving home" until the full sequence has been completed and one arrives at home. All events used in this study were of the accomplishment/filter type, with duration and obligatory temporal end points.

Each action was described in either the simple or the progressive past tense, as in, "The man (made/was making) a fire." For each sentence pair, two pictures were drawn by the first author and a research assistant. One picture depicted the action in an intermediate stage (the man putting logs in the fireplace), and the other depicted the action at a completed or near-completed stage (the fire is present and the man is still holding the match; see the Appendix).

Across four lists, each event item occurred equally often as a perfective sentence and as an imperfective sentence, with position of the two pictures on the screen counterbalanced across lists as well. Each participant saw only one list. Thus, participants saw only one version of each experimental item. Because perfective aspect denotes a completed action, on trials with a perfective sentence, the completed picture would be considered a "match," and the intermediate picture would be considered a "mismatch." Because imperfective aspect denotes an in-progress action, on trials with an imperfective sentence, the completed picture would be considered a "mismatch," and the intermediate picture would be considered a "match."

Procedure. Instructions displayed on a computer screen directed participants to read sentences and judge which of two subsequently presented pictures matched the sentence best. On a given trial, a fixation cue $(* * * \operatorname{READY} * * *)$ appeared in the middle of the screen for $1 \mathrm{sec}$. Next, a sentence was presented in the middle of the screen for $1.5 \mathrm{sec}$ before the two pictures appeared side by side above the sentence. The participants were to press the labeled left or right key on the keyboard to indicate which picture matched the sentence best. The stimuli were presented and the responses were recorded with PsyScope software (Cohen, MacWhinney, Flatt, \& Provost, 1993) on a Power Macintosh 7200/120 with an Apple 15-in. monitor.

\section{Results and Discussion}

One of the 26 items was removed before the analyses were conducted, because the picture file was corrupted on most of the participant runs. In addition, one item yielded poor speed and accuracy responses in all conditions of Experiments 1 and 2. Upon further review, this picture was considered to be a poor representation of the described event and the item was removed from all three experiments before the analyses were conducted.

The dependent measure in this experiment was the percentage of "matching" pictures chosen. The matching picture for a perfective sentence was the completed picture; the matching picture for an imperfective sentence was the in-progress picture. Although list was included as a factor in the analyses (Pollatsek \& Well, 1995; Raaijmakers, Schrijnemakers, \& Gremmen, 1999), effects for the list variable will not be reported, given their lack of theoretical relevance. Analyses were conducted with both variability due to subjects and variability due to items in the error term. These analyses are indicated by the subscripts 1 and 2, respectively. The alpha level in all analyses reported below was .05 .

The results show a clear effect of aspect, manifest in the overall match/mismatch analysis of variance (ANOVA), 
collapsed across perfective and imperfective sentences, with list as a between-subjects factor. Participants chose the matching picture (completed after having read perfective, and in progress after having read imperfective) in $66 \%$ of the trials $(S D=13 \%)$. This is more than chance would predict $\left[F_{1}(1,16)=40.61, M S_{\mathrm{e}}=6,504 ; F_{2}(1,20)=\right.$ 63.75, $\left.M S_{\mathrm{e}}=4,337\right]$. However, when the aspect sentence variable is introduced and both perfective and imperfective aspects were examined separately, an interesting pattern emerged. A within-subjects ANOVA for two levels of aspect (perfective/imperfective) with list as the betweensubjects factor showed that the match effect was significantly greater for the perfective than for the imperfective sentences $\left[F_{1}(1,16)=5.06, M S_{\mathrm{e}}=743 ; F_{2}(1,20)=7.22\right.$, $\left.M S_{\mathrm{e}}=673\right]$. Participants chose the matching picture (completed picture) on $76 \%$ of the perfective trials $(S D=$ $21 \%$ ), but chose the matching picture (in-progress picture) on only $56 \%$ of the imperfective trials $(S D=28 \%)$. Single-sample $t$ tests showed that only when participants read the perfective sentences were they more likely to choose the matching picture than would be predicted by chance $\left[F_{1}(1,16)=28.00, M S_{\mathrm{e}}=246 ; F_{2}(1,20)=36.40\right.$, $\left.M S_{\mathrm{e}}=216\right]$. When participants read the imperfective sentences, they chose the in-progress and completed pictures equally often (both $F_{\mathrm{S}}<1$ ). Hence, the overall match effect, indicating that readers are sensitive to aspectual cues, seems to have been due solely to the perfective sentences.

The overall match effect is consistent with the findings of Morrow (1985a, 1990). In those studies, the perfective and imperfective aspects were used to describe characters moving from room to room. The participants inferred that the character was in the target room more often when the perfective was used, and still en route when the imperfective was used. However, in our Experiment 1 as well as in Morrow's experiments, forced choice tasks were employed. We therefore conducted a second experiment to assess whether these differential representations are formed routinely, or only when participants are prompted to do so, as Experiment 1 might have demanded.

\section{EXPERIMENT 2}

In Experiment 2, we employed response time, a more implicit measure of representation availability than forced choice. The participants read the same sentences as in Experiment 1, but they were then presented with a single picture. They were required to make a yes/no decision as to whether or not the picture matched the sentence. All experimental event items were intended to elicit a "yes" decision, but the response latencies were expected to be longer for the ongoing pictures when they were preceded by a perfective sentence than when they were preceded by an imperfective sentence. Likewise, the response latencies were expected to be longer for the completed pictures when they were preceded by an imperfective sentence than when they were preceded by a perfective sentence. In light of the results from Experiment 1, we expected that the perfective sentences would show a greater match/ mismatch difference than would the imperfective sentences. Therefore, the response times were analyzed according to a 2 (perfective/imperfective sentence) $\times 2$ (match/mismatch picture) ANOVA design.

\section{Method}

Participants. Fifty-two undergraduate students enrolled at Florida State University participated in the experiment. Participation was part of a course requirement in psychology classes. All participants were native English speakers.

Materials. The same 26 simple/progressive sentence pairs and ongoing/completed picture pairs from Experiment 1 were used in this experiment. This time, however, only one picture was paired with each sentence. This 2 (perfective/imperfective sentence) $\times 2$ (in-progress picture/completed picture) design yielded two match and two mismatch versions for each item: match = perfective + completed picture, or imperfective + in-progress picture; mismatch $=$ perfective + in-progress picture, or imperfective + completed picture. Four lists were created such that each event item occurred in each of the four possible conditions, counterbalanced across participants. In addition, 26 filler sentences and nonmatching pictures were constructed so that there would be equal numbers of "yes" and "no" responses. These filler sentences conformed to the same simple or progressive past tense format as that of the experimental sentences, but the subsequent pictures were blatantly unrelated to the sentences (e.g., "The girl was tying her shoe," followed by a picture of a man using a laptop). Thus, the fillers were "nonmatches," requiring a "no" response. This is in contrast to experimental "mismatches," which required a "yes" response, but were hypothesized to be slower than the "yes" responses for experimental "matches."

Procedure. Instructions were displayed on a computer screen, explaining that the participant was to read sentences and judge whether a subsequently presented picture did or did not match the sentence. On a given trial, a fixation cue (***READY***) appeared in the middle of the screen for $1 \mathrm{sec}$. Next, a sentence was presented in the middle of the computer screen. The participants were instructed to read the sentence and press the spacebar once finished. Upon the spacebar press, a single picture appeared above where the sentence had been. The participants were instructed to press the labeled YES or NO keys on the keyboard as fast as they could to indicate whether or not the picture matched the sentence. The stimuli were presented and the responses were recorded with PsyScope software (Cohen et al., 1993) on a Power Macintosh 7200/120 with an Apple 15-in. monitor.

\section{Results and Discussion}

The item considered to be a poor representation in Experiment 1 was also excluded from the analyses in Experiment 2 . In addition, the data from 6 of the 52 participants were removed because of their extremely long response times and inaccurate responses, which indicated that they had not performed the task correctly. These 6 participants seemed to be meticulously studying the pictures, looking closely for inconsistencies, rather than just looking at them briefly to decide whether the picture generally matched the preceding sentence as they had been instructed. In the remaining participants' data, responses over $1,700 \mathrm{msec}$ and responses plus or minus 2.5 standard deviations from the participant's condition mean were removed. These responses consisted of less than $5 \%$ of the data. Only correct ("yes") responses were used 5 in the analyses. All experimental items in both the 
match and the mismatch conditions were intended to elicit a "yes" decision, but the response latencies were expected to be longer when the aspect of the preceding sentence did not match the completion status of the picture.

The participants provided "yes" responses on $95 \%$ of the trials in both the match and the mismatch conditions. This confirms that the participants were following instructions and evaluating the pictures on a global level, generally responding "no" only to the filler items (98\% "no" responses on fillers). The identical accuracy scores across the match and mismatch conditions in experimental items suggest that the manipulation was sufficiently subtle and that the participants probably did not explicitly notice the in-progress/completed aspect manipulation in the pictures and sentences.

The means and standard deviations from Experiment 2 are displayed in Table 1 . The interaction between match and aspect was significant by participants, but did not reach significance in the item analysis $\left[F_{1}(1,42)=6.25\right.$, $\left.M S_{\mathrm{e}}=5,561 ; F_{2}(1,21)=2.38, M S_{\mathrm{e}}=12,205, p<.14\right]$. Just as in Experiment 1, when each aspect construction was examined separately, the participants did not appear to be sensitive to the imperfective aspect cues. Only when the participants read the perfective sentences were they faster to respond to the matching picture than to the mismatching picture $\left[F_{1}(1,42)=10.46, M S_{\mathrm{e}}=5,253\right.$; $\left.F_{2}(1,21)=4.80, M S_{\mathrm{e}}=8,221\right]$. When the participants read the imperfective sentences, they responded equally fast to the in-progress and completed pictures (both $F_{\mathrm{S}}<1$ ).

The ANOVA also revealed a marginal main effect of match, indicating that participants were faster to respond to matching pictures (completed when having read perfective, and in-progress when having read imperfective) than to mismatching pictures (completed when having read imperfective, and in-progress when having read perfective) $\left[F_{1}(1,42)=3.15, M S_{\mathrm{e}}=6,670, p<.09 ; F_{2}(1,21)=\right.$ $\left.1.83, M S_{\mathrm{e}}=6,697, p<.20\right]$. Thus, the overall effect of match indicates that the participants were indeed sensitive to aspectual cues. However, as the interaction demonstrates, this marginal main effect was largely due to the perfective sentences.

\section{EXPERIMENT 3}

In the previous two experiments, we intended to learn something about the way in which aspectual information is represented in situation models for simple sentences by choosing a task that required subjects to compare these already constructed situation models with actual pictures of the events. In Experiment 3, however, the goal was different. Here the intent was to determine whether aspectual information in pictures of events can facilitate the subsequent construction and evaluation of a situation model when one is presented with a sentence. Toward this aim, in Experiment 3 we reversed the order of sentences and pictures so that a picture was presented first and the associated sentence followed. In this experiment, the dependent measure was the time to read the sentence and make a judgment about the relatedness to the previously presented picture.

\section{Method}

Participants. Sixty-four undergraduate students enrolled at Florida State University participated in the experiment. Participation was part of a course requirement in psychology classes. All participants were native English speakers.

Materials. Twenty-eight simple/progressive sentence pairs and ongoing/completed picture pairs were used in this experiment. Twenty-six of these were from Experiments 1 and 2, and 2 new items were added to the set. The experimental sentences had an average length of 5.86 words $(S D=1.08)$. Otherwise, the four lists of experimental items and fillers were the same as in Experiment 2.

Procedure. Instructions were displayed on a computer screen, explaining that the participant was to look at pictures and read sentences, judging whether or not the sentence was related to the picture just presented. On a given trial, a cue appeared in the middle of the screen, directing participants to press the spacebar when they were ready for the next picture. As soon as the spacebar was pressed, a picture appeared for $3 \mathrm{sec}$. Next, a sentence was presented in the middle of the computer screen. The participants were instructed to read the sentence and press the specially labeled YES or NO keys on the keyboard to indicate whether or not the sentence was related to the picture as soon as they had finished reading the sentence. The stimuli were presented and the responses were recorded with E-Prime software (Schneider, Eschman, \& Zuccolotto, 2002) on a Dell PC, with a Trinitron 19-in. monitor.

\section{Results and Discussion}

The item considered to be a poor representation in Experiments 1 and 2 was excluded from the analyses in Experiment 3 . In addition, the data from 4 of the 64 participants were removed because of their extremely long response times and inaccurate responses, which indicated that they had not performed the task correctly. These 4 participants seemed to have been studying the sentences rather than simply reading them, and to have been responding on the basis of some other criterion than that given in the instructions. In the remaining participants' data, responses over 4,000 msec and responses

Table 1

Means and Standard Deviations (in Milliseconds) for the Picture Verification Task Following the

Perfective and Imperfective Sentence Presentations (Experiment 2), and for the Sentence Verification Task Following the Completed and In-Progress Picture Presentations (Experiment 3)

\begin{tabular}{|c|c|c|c|c|c|c|c|c|c|c|c|c|}
\hline & \multicolumn{6}{|c|}{ Match } & \multicolumn{6}{|c|}{ Mismatch } \\
\hline & \multicolumn{2}{|c|}{ Overall } & \multicolumn{2}{|c|}{ Perfective } & \multicolumn{2}{|c|}{ Imperfective } & \multicolumn{2}{|c|}{ Overall } & \multicolumn{2}{|c|}{ Perfective } & \multicolumn{2}{|c|}{ Imperfective } \\
\hline & $M$ & $\overline{S D}$ & $M$ & $\overline{S D}$ & $M$ & $\overline{S D}$ & $M$ & $\overline{S D}$ & $M$ & $\overline{S D}$ & $M$ & $S D$ \\
\hline Experiment 2 & 690 & 205 & 674 & 207 & 710 & 221 & 709 & 210 & 719 & 224 & 698 & 212 \\
\hline Experiment 3 & 1,238 & 376 & 1,181 & 345 & 1,294 & 399 & 1,267 & 363 & 1,265 & 371 & 1,270 & 358 \\
\hline
\end{tabular}


plus or minus 2 standard deviations from the participants' condition means were removed. These responses consisted of less than $5 \%$ of the data. Only correct ("yes") responses were used in the analyses. All experimental items in both the match and the mismatch conditions were intended to elicit a "yes" decision, but the response latencies were expected to be longer when the completion status of the picture did not match the aspect of the subsequent sentence.

The participants provided "yes" responses on $95 \%$ and $94 \%$ of the trials in the match and mismatch conditions, respectively. There was no significant difference between these accuracy scores. This confirms that the participants were following instructions and evaluating the sentences on a global level, generally responding "no" only to the filler items (99\% "no" responses on fillers). Just as in Experiment 2, the similar accuracy scores across the match and mismatch conditions in experimental items suggest that the participants probably did not explicitly notice the in-progress/completed aspect manipulation in the pictures and sentences.

The means and standard deviations from Experiment 3 are displayed in Table 1. As predicted, there was a significant interaction between match and aspect (by subjects only) $\left[F_{1}(1,56)=10.76, M S_{\mathrm{e}}=16,404 ; F_{2}(1,23)=\right.$ 2.30, $\left.M S_{\mathrm{e}}=34,092, p<.15\right]$. Just as in Experiment 1, when each aspect construction was examined separately, the imperfective aspect was not as constraining as the perfective aspect. Only on the perfective sentences were participants faster to respond if they had previously viewed the completed pictures than if they had viewed the in-progress pictures $\left[F_{1}(1,56)=13.50, M S_{\mathrm{e}}=15,632\right.$; $\left.F_{2}(1,23)=4.37, M S_{\mathrm{e}}=24,086\right]$. However, whether participants viewed the in-progress or completed pictures, they responded to the subsequent imperfective sentences equally fast (both $F_{\mathrm{S}}<1$ ).

\section{GENERAL DISCUSSION}

We investigated the extent to which readers use aspectual cues when the construct situation models. In Experiment 1, participants read sentences and chose which of two pictures matched each sentence better. The sentences used either simple (perfective) or progressive (imperfective) aspect in the past tense to describe agents engaging in events. Participants chose the picture of the event at a completed stage more often than at an inprogress stage when the preceding sentence used the perfective aspect. However, after reading the imperfective aspect sentences, participants chose completed and inprogress pictures equally often, indicating that the imperfective aspect does not seem to constrain readers' representations to an unfinished stage of the described event.

A very similar pattern of results was observed in Experiment 2 . In this experiment, the same perfective and imperfective aspect sentences were used as well as the same completed and in-progress pictures. However, after each sentence, only one picture was presented and par- ticipants made a speeded response as to whether or not that picture matched the preceding sentence. They were faster to respond "yes" to completed pictures than to inprogress pictures after having read the perfective aspect sentences. Just as in Experiment 1, participants' representations did not seem to be constrained by the imperfective aspect manipulation. They did not respond any faster to in-progress pictures than to completed pictures when the preceding sentence used the imperfective aspect.

In Experiment 3, the pictures were presented first, and the sentences followed. Again, the perfective sentences showed a reduction in response time following the completed pictures relative to the in-progress pictures, but the imperfective sentences were responded to equally fast regardless of which picture was previously presented.

This pattern of results indicates that readers do in fact use aspectual cues when they construct situation models. Given the forced choice data from Experiment 1 as well as the response latency data from Experiments 2 and 3, it is clear that the representations formed are reflective of the aspectual cues in the sentences. However, this appears to be true only for the perfective (simple past tense) trials, and not the imperfective (progressive past tense) aspect trials. Why does only one half of this aspectual dichotomy effectively constrain readers' situation model representations? We will now consider three possible explanations for these results.

First, a nontheoretical explanation must be addressed. ${ }^{1}$ It is possible that the difference between the imperfective and perfective aspects with respect to temporal constraints is driven entirely by participants' interpretations of the pictures themselves. The incomplete pictures are presumably unambiguous with respect to the interpretation of the temporal status of the events (i.e., it is in progress). However, the completed pictures may be ambiguous with respect to the temporal status of the events. Viewers could interpret the completed pictures as still ongoing rather than entirely finished. If so, this picture interpretation would explain the finding that imperfective sentences allow either picture, whereas perfective sentences only favor the completed picture.

This idea was evaluated by conducting a separate experiment in which 24 participants viewed one version (in progress or completed) of each picture from the three experiments. Each of these 27 pictures appeared with a simple present tense event heading (e.g., "paint picture") at the top of the page and a time line below the picture. The participants were told that the dot at the beginning (left) of the time line marked the onset of the event and the dot at the end (right) of the time line marked the completion of the event. The participants were instructed to look at each of the 27 pictures appearing on separate pages and determine at what point in time the picture displayed the event described by the heading. Then the participants were to place an " $X$ " on the time line below the picture at the specific stage of completion at which they perceived the event to be depicted.

The specific point marked by the participant on the 43-mm time line was measured for each item, and the 
mode score across participants was calculated. This measure was chosen to remove the influence of the few idiosyncratically "early" ratings from the skewed distribution of time-line ratings for the completed pictures. As expected, the ratings for in-progress items indicated that the pictures were perceived as early to midway through the event $($ mode $=37 \%$ completed , median $=40 \%$ completed), whereas the ratings for completed items indicated that the pictures were perceived on or near the end point $($ mode $=95 \%$ completed, median $=93 \%$ completed) [medians: $t(26)=14.47, p<.0001]$. However, given that near the end point is still problematic, we reran the item analyses for Experiments 1 and 2, excluding any items that did not produce a mode of at least $95 \%$, which touches the end point of the time line. Six of the 27 completed items were removed from the set (5 from the set of 25 items used in Experiments 1 and 2). These 6 items were the following events: digging a hole, knitting a sweater, mowing the lawn, running up the stairs, setting the table, and shaving his face. Upon reviewing these 6 completed pictures, we determined that the agent could have been perceived as continuing to dig, knit, and so on more so than in the remaining completed pictures.

Imperfective analyses were rerun to investigate whether the participants' lack of discrimination between completed and in-progress pictures for the imperfective sentences was due to ambiguity of the completed pictures (possibly perceived as in-progress). However, the item analysis for the imperfective items in Experiments 1,2, and 3 showed the same nonsignificant effects as those reported in the Results and Discussion sections above [Experiment 1, $F(1,15)=1.15, M S_{\mathrm{e}}=263, p>.3$; Experiment $2, F(1,16)<$ $1, M S_{\mathrm{e}}=11,109, p>.4$; Experiment $3, F(1,17)<1$, $\left.M S_{\mathrm{e}}=27,647, p>.9\right] .{ }^{2}$ Table 2 displays the new means for perfective and imperfective pictures/sentences for Experiments 1, 2, and 3 with the six items removed on the basis of mode scores below $95 \%$ (41 mm). To be absolutely certain that potential ambiguity in the finality of the completed pictures did not drive the lack of effect in the imperfective sentences, the more conservative median scores from the time-line ratings were also ex- amined, and scores below $95 \%(41 \mathrm{~mm})$ were removed. If the lack of an effect for the imperfective sentences was due to the completed pictures' being perceived as ongoing, constraining the item set to the completed pictures that were definitely perceived as finished should yield a shift in means such that a match effect would begin to emerge in the imperfective sentences. Although power for detecting an effect would be low in this case with so many items removed, it is clear from inspecting the new means that the scores do not shift to support this nontheoretical explanation. The participants were not more likely to choose the ongoing picture/sentence over the completed picture/sentence even for items in which the completed pictures were rated as clearly depicting end points of the events. Thus it must be something about the grammatical construction of the imperfective aspect rather than misperceptions about the completed pictures that causes this ambiguity.

Before we address the two theoretical explanations, it should be stated that the present study does not provide discriminating evidence on whether representations formed during language comprehension are perceptual simulations of the described events, as theorized by Barsalou (1999), or amodal, propositional representations of the events (Carpenter \& Just, 1992; Kintsch, 1988). Although the results of the three experiments are consistent with the perceptual simulation theory, they could also be accounted for in terms of propositional representations. Nevertheless, because of the growing body of evidence for perceptual simulations (Glenberg \& Kaschak, 2002; Klatzky, Pellegrino, McCloskey, \& Doherty, 1989; Morrow \& Clark, 1988; Pecher, Zeelenberg, \& Barsalou, 2003; Stanfield \& Zwaan, 2001; Zwaan et al., 2002), we will discuss the results of the present study in terms of simulations rather than propositional representations.

One possible explanation for our findings is that not all comprehenders represent the imperfective sentences at the same intermediate stage of completion. The items used in the present study were events that comprised multiple stages toward the events' completion. Perhaps each reader simulated the painting in "The girl was painting a

Table 2

Means and Standard Deviations (in Percentages for the Picture-Choosing Task in Experiment 1, in Milliseconds for the Picture Verification Task in Experiment 2) Following the Perfective and Imperfective Sentence Presentations, and for the Sentence Verification Task Following the Completed and In-Progress Picture Presentations (Experiment 3) After Six Items With Mode Time-Line Ratings Below 41 mm Were Removed and After Fourteen Items With Median Time-Line Ratings Below $41 \mathrm{~mm}$ Were Removed

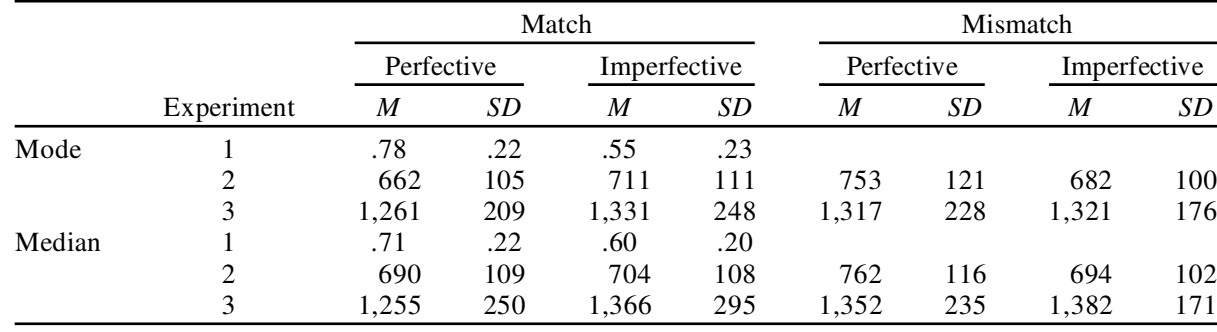


picture" at a different stage of completion. In this case, it would be impossible to present a picture that exactly matched the intermediate stage that was foregrounded in each individual reader's representation. Even in Experiment 3 , when the picture was presented first, the inprogress picture might not have matched the intermediate stage that the reader spontaneously simulated upon reading the subsequent sentence. In contrast, the final stage of an event is much better defined, so this would not have been a problem with the perfective sentences. It is much easier to converge on a representation for the end of an event across readers, and presumably the pictures used in the present study captured this end point sufficiently. In this case, we would expect that the imperfective sentencepicture matches would not show the same benefit that the perfective sentence-picture matches would show.

Alternatively, it is possible that all readers form similar representations of imperfective sentences, but that they represent the internal structure of the event dynamically, simulating each stage of the event sequentially as the stages would be experienced. In this case, both of the pictures that were presented in these experiments would have matched the representations because both intermediate as well as end stages of the event would have been included in the simulation. Here, we would expect that both the match and the mismatch conditions for the imperfective would show the same speed benefit as would the match condition for the perfective. In fact, we might even expect that the imperfective mismatch would be faster than the imperfective match and just as fast as the perfective match, if the design allowed comprehenders enough time to reach the end point of their simulations before the picture was presented. If the dynamic simulation played to completion, the end state would be more active than any middle state, so there would be a match between imperfective sentences and completed pictures. However, linguistic theory dictates that the imperfective representations should not include the end point, because a subsequent sentence might actually describe something from the middle of the event, or something that interrupted the event so that the end point was never reached.

The data from Experiments 2 and 3 demonstrate that the imperfective sentence-picture matches do not show the same benefit as do the perfective sentence-picture matches (or an equal mismatch benefit). The condition means for Experiments 2 and 3 provide evidence that it is a match benefit occurring in the perfective rather than some deficit that occurs in the imperfective. In Experiment 2 , the average response latency for the perfective match condition was significantly shorter (by subjects, but not by items) than that for the imperfective match condition $\left[F_{1}(1,42)=4.73, M S_{\mathrm{e}}=7,691 ; F_{2}<1\right]$. However, there is no significant difference between the mismatch condition response latency for perfective and imperfective (both $F_{\mathrm{S}}<1$ ). Likewise, in Experiment 3, the average response latency for the perfective match condition was significantly shorter than that for the imperfective match condition $\left[F_{1}(1,56)=13.92, M S_{\mathrm{e}}=27,510\right.$;
$\left.F_{2}(1,23)=7.50, M S_{\mathrm{e}}=22,537\right]$. However, there is no significant difference between the mismatch condition response latency for perfective and imperfective (both $\left.F_{\mathrm{S}}<1\right)$. In both of these cases, it should be pointed out that the comparison was based on responses to two slightly different pictures (Experiment 2) and responses to two slightly different sentences (Experiment 3, with perfective sentences being shorter). However, it is clearly evident in the means for both experiments that the match condition for the perfective produced significantly shorter latencies than did any of the other three conditions. Thus, it seems plausible that a match benefit is responsible for the pattern of results in the perfective rather than some deficit that occurs in the imperfective. This is consistent with the idea that different readers are representing the imperfective sentences at various intermediate stages of completion. However, this hypothesis will require further investigation.

Finally, Magliano and Schleich (2000) presented data in their Experiments 1 and 2 that indicate that the imperfective aspect is interpreted as ongoing rather than completed, which is seemingly inconsistent with the present findings. This can potentially be explained by differences in the type of information contained in the perfective and imperfective aspects and by how the methodologies differ between the two studies. Magliano and Schleich presented stories containing a critical sentence described in the imperfective or perfective aspect. Participants responded to yes/no questions about whether the event described in the critical sentence was still ongoing. The participants responded more often that imperfective events were ongoing, whereas the present study suggests that events could be perceived as either ongoing or completed. However, it is possible that the pictures employed in the present study provided a richer set of cues for assessing the event representations for perfective and imperfective sentences. Thus, participants perceived the activity in the completed pictures as finished, yet many of the other features present in the completed picture were still relevant to the ongoing event. For instance, the location, agent, and typically the instrument were each available in the completed picture and might have helped facilitate the ongoing event representation despite the fact that the event itself was depicted as completed.

Each of these event features (location, agent, instrument) has been shown to be more available when events are described in the imperfective aspect rather than the perfective aspect (Carreiras et al., 1997; Ferretti, 2000; Truitt \& Zwaan, 1997). These features help define imperfective event representations, whereas they do not seem to influence perfective event representations, which is consistent with the idea that the imperfective aspect allows internal access to the event, whereas the perfective aspect does not. This may explain the difference between the results of the present study, which incorporated visual presentation of event features, and those of Magliano and Schleich's (2000) study, which employed an explicit decision about duration. Thus, the results are not incom- 
patible, given that participants in the two studies were accessing separate types of information about verb aspect. Both types of information are important and informative. The imperfective aspect does convey information about duration, specifically that an event is ongoing. However, it also incorporates the heightened importance of event features. The imperfective aspect acts as a linguistic cue for constructing an event representation that foregrounds the location, agent, and instrument of the ongoing event, whereas the perfective aspect directs the reader to construct an event representation that foregrounds the resultant state of the completed event. Both types of pictures in the present study are compatible with the way in which the imperfective aspect is represented (foregrounded features), whereas only the completed pictures are compatible with the way in which perfective events are represented (resultant state). Therefore, although the completed pictures are perceived as finished events, they may incorporate more foregrounded features than a typical perfective representation does, and thus they are compatible with both imperfective and perfective representations.

In addition, another difference between the study by Magliano and Schleich (2000) and the present study is that the former employed a task in which participants read whole stories. One function of the imperfective aspect, as described in the introduction, is to keep events available in an ongoing state, so that subsequently described events may occur while the current is still in progress. In this case, comprehenders would not include the end point in their representations. Because Magliano and Schleich used aspect sentences embedded in stories, participants were more likely to expect subsequent events to be described. Thus, this feature of the imperfective might have had a greater influence in Magliano and Schleich's study than in the present study, in which single sentences were presented in isolation. In the present study, participants did not have the usual expectation about upcoming information and therefore may have been more inclined to include the end point in their simulations. If the experimental sentences used here were embedded into larger stories, we would expect to find that the imperfective representations would be more constrained to an in-progress state.

\section{CONCLUSION}

The present study shows a clear effect of aspectual cues on readers' situation models for single sentences. However, the perfective aspect seemed to impose a greater constraint on these representations than did the imperfective aspect. The pattern of results suggests that the imperfective trials lacked the match benefit that the perfective trials demonstrated, because of the inconsistency across participants in their imperfective event representations. In addition, the results suggest that the types of information represented in imperfective and perfective event representations are inherently different, with imperfective events foregrounding event features, whereas perfective events foreground a resultant event state. Because the perfective aspect does not allow access to the internal structure and the features of an event, event availability has been difficult to assess in the perfective aspect. In the present study, we used picture presentations to provide a clear demonstration of the availability and constraints of the perfective aspect.

The topic of verb aspect must be empirically addressed in future research so that it can yield a better understanding of how the imperfective and perfective aspects affect situation models during comprehension. The present study furthers our understanding of how subtle grammatical cues such as verb aspect influence the representations formed when we read or hear language. However, this is only the beginning of our pursuit to understand how situation models are constructed from our complicated linguistic code.

\section{REFERENCES}

Bar sal ou, L.W. (1999). Perceptual symbol systems. Behavioral \& Brain Sciences, 22, 577-660.

Car pent er, P. A., \& Ju st, M. A. (1992). A capacity theory of comprehension: Individual differences in working memory. Psychological Review, 99, 122-149.

Carreir as, M., Carriedo, N., Al onso, M. A., \& Fernández, A. (1997). The role of verb tense and verb aspect in the foregrounding of information during reading. Memory \& Cognition, 25, 438-446.

$\mathrm{Cl}$ ark, H. H., \& Chase, W. G. (1972). On the process of comparing sentences against pictures. Cognitive Psychology, 3, 472-517.

Cohen, J. \{D.\}, MacWhinney, B., Fl at t, M., \& Provost, J. (1993). PsyScope: An interactive graphic system for designing and controlling experiments in the psychology laboratory using Macintosh computers. Behavior Research Methods, Instruments, \& Computers, 25, 257-271.

Comr ie, B. (1976). Aspect. Cambridge: Cambridge University Press. Dowt y, D. R. (1986). The effects of aspectual class on the temporal structure of discourse: Semantics or pragmatics? Linguistics \& Philosophy, 9, 37-61.

Fer ret t i, T. R. (2000). Situation schemas, thematic roles and grammatical morphemes. Unpublished doctoral dissertation, University of Western Ontario.

Givón, T. (1992). The grammar of referential coherence as mental processing instructions. Linguistics, 30, 5-55.

Gl en berg, A. M., \& Ka sch ak, M. P. (2002). Grounding language in action. Psychonomic Bulletin \& Review, 9, 558-565.

Gl enber g, A. M., Robert son, D. A., Jan sen, J. L., \& JohnsonGl en ber g, M. C. (1999). Not propositions. Journal of Cognitive Systems Research, 1, 19-33.

Hopper, P. J. (1979). Aspect and foregrounding in discourse. In T. Givón (Ed.), Syntax and semantics: Vol. 12. Discourse and syntax (pp. 213-241). New York: Academic Press.

Kint sch, W. (1988). The role of knowledge in discourse comprehension: A construction-integration model. Psychological Review, 95, 163-182.

$\mathrm{Kl}$ at zky, R. L., Pel 1 egr ino, J. W., McCl oskey, B. P., \& Doher ty, S. (1989). Can you squeeze a tomato? The role of motor representations in semantic sensibility judgments. Journal of Memory \& Language, 28, 56-77.

Magl iano, J. P., \& Schl eich, M. C. (2000). Verb aspect and situation models. Discourse Processes, 29, 83-112.

Morrow, D. G. (1985a). Prepositions and verb aspect in narrative understanding. Journal of Memory \& Language, 24, 390-404.

Mor row, D. G. (1985b). Prominent characters and events organize narrative understanding. Journal of Memory \& Language, 24, 304-319.

Morrow, D. G. (1990). Spatial models, prepositions, and verb-aspect markers. Discourse Processes, 13, 441-469.

Morrow, D. G., \& Cl ar k, H. H. (1988). Interpreting words in spatial descriptions. Language \& Cognitive Processes, 3, 275-291. 
Pecher, D., Zeel en berg, R., \& Barsal ou, L. W. (2003). Verifying different-modality properties for concepts produces switching costs. Psychological Science, 14, 119-124.

Poll at sek, A., \& Wel 1, A. D. (1995). On the use of counterbalanced designs in cognitive research: A suggestion for a better and more powerful analysis. Journal of Experimental Psychology: Learning, Memory, \& Cognition, 21, 785-794.

Raaijmakers, J. G. W., Schrijnemakers, J. M. C., \& Gremmen, F. (1999). How to deal with "the language-as-fixed-effect fallacy": Common misconceptions and alternative solutions. Journal of Memory \& Language, 41, 416-426.

Schneider, W., Eschman, A., \& Zuccol ot t o, A. (2002). E-Prime reference guide. Pittsburgh: Psychology Software Tools.

St anfiel d, R. A., \& Zwaan, R. A. (2001). The effect of implied orientation derived from verbal context on picture recognition. Psychological Science, 12, 153-156.

t er Meul en, A. G. B. (1995). Representing time in natural language: The dynamic interpretation of tense and aspect. Cambridge, MA: MIT Press.

Truit t, T. P., \& Zwa an, R. A. (1997, November). Verb aspect affects the generation of instrument inferences. Paper presented at the 38th Annual Meeting of the Psychonomic Society, Philadelphia.

van Dijk, T. A., \& Kint sch, W. (1983). Strategies of discourse comprehension. New York: Academic Press.

Vendl er ,Z. (1957). Verbs and times. PhilosophicalReview, 66, 143-160.
Vendl er, Z. (1967). Linguistics in philosophy. Ithaca, NY: Cornell University Press.

Zwaan, R. A., \& Radvansky, G. A. (1998). Situation models in language comprehension and memory. Psychological Bulletin, 123, 162-185.

Zwa an, R. A., St anfiel d, R. A., \& Yaxl ey, R. H. (2002). Language comprehenders mentally represent the shapes of objects. Psychological Science, 13, 168-171.

\section{NOTES}

1. Thanks to Art Glenberg, Joe Magliano, and an anonymous reviewer for pointing out this possible alternative explanation.

2 . The perfective item analyses were also rerun after the six problematic items were removed. Results from Experiments 1, 2, and 3 showed the same significant effects for the perfective aspect as those reported in the results sections, with the exception that the Experiment 3 ANOVA was now only marginally significant [Experiment $1, F(1,15)=18.34$, $M S_{\mathrm{e}}=259, p<.01$; Experiment $2, F(1,16)=5.84, M S_{\mathrm{e}}=8,856, p<.03$; Experiment $\left.3, F(1,17)=3.62, M S_{\mathrm{e}}=29,858, p<.075\right]$. The ability to detect effects in these perfective conditions suggests that we had enough power to detect similar effects in the respective imperfective conditions if such effects existed. Power analyses for the new subset of items support this claim, especially for Experiments 1 and 2 (Experiment 1 , power $=.97$; Experiment 2, power $=.69$; Experiment 3 , power $=.30$ ).

\section{APPENDIX \\ Sample Pictures Depicting Events (Speeding/Sped Through an Intersection, Making/Made a Fire) as In Progress and Completed}

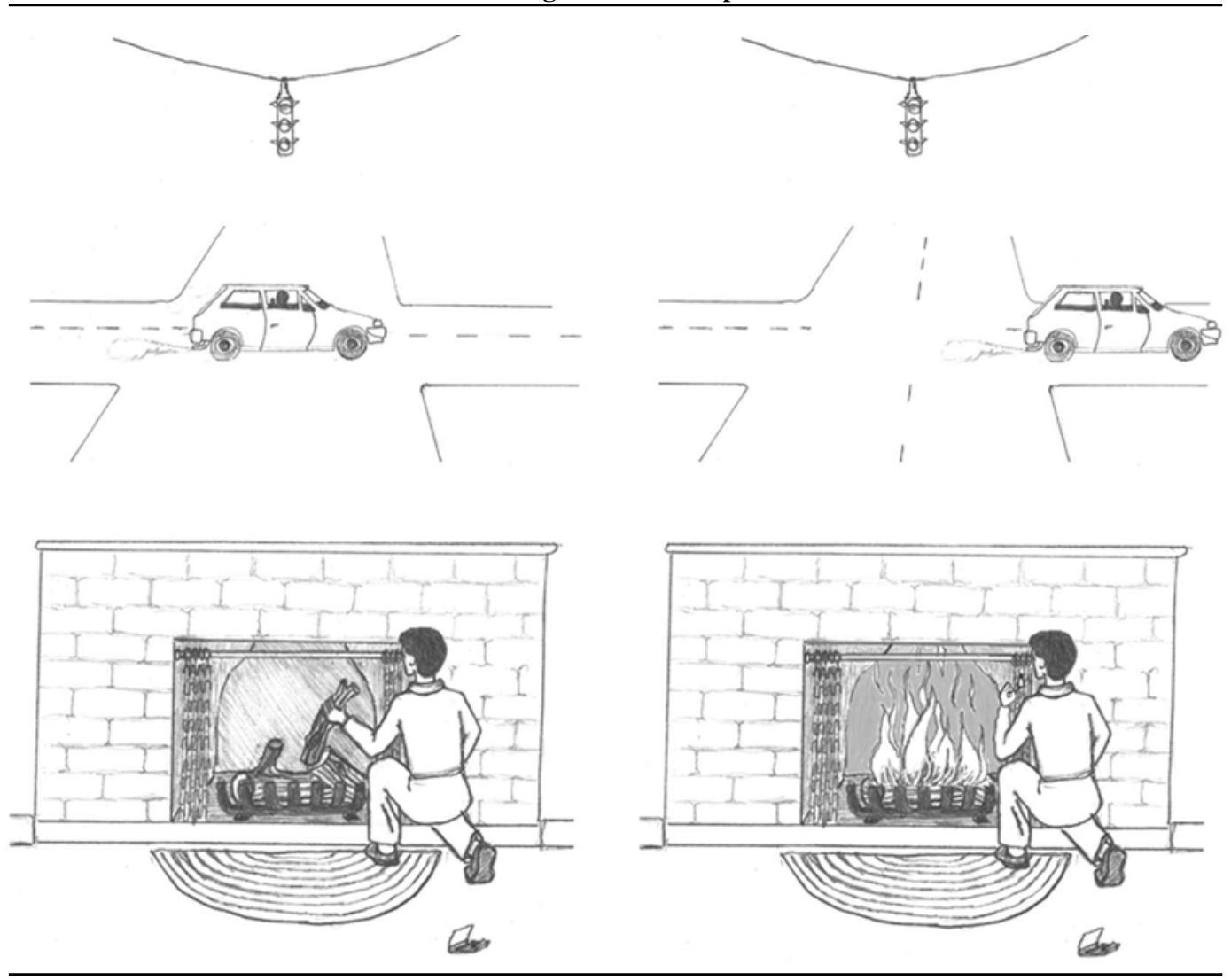

(Manuscript received June 4, 2002;

revision accepted for publication March 28, 2003.) 